\title{
Influence of Peak Wavelengths on Properties of Mixed-LED White-Light Sources
}

\author{
Snjezana Soltic and Andrew N. Chalmers \\ EET School, Manukau Institute of Technology, Private Bag 94-006, Manukau 2241, New Zealand
}

Correspondence should be addressed to Snjezana Soltic, ssoltic@manukau.ac.nz

Received 3 October 2010; Accepted 6 December 2010

Academic Editor: Xian Cao

Copyright (C) 2010 S. Soltic and A. N. Chalmers. This is an open access article distributed under the Creative Commons Attribution License, which permits unrestricted use, distribution, and reproduction in any medium, provided the original work is properly cited.

The purpose of this investigation is to quantify the influence of the peak wavelength shifts in commercially available LEDs on the characteristics of the mixed-LED white-light sources. For this purpose, a tetrachromatic spectrum was optimized and then subjected to deviations in the peak wavelengths. A total of 882 combinations of peak wavelength values were evaluated, and the results are reported in terms of correlated colour temperature, colour-rendering properties, and radiant luminous efficacy. The results show that there can be significant changes in the characteristics of the source under these conditions. Such changes are highly likely to present problems when dealing with applications where an effective and accurate white-light source is important.

\section{Introduction}

White light can be produced by additively combining the outputs of multiple monochromatic light-emitting diodes (LEDs) [1-6]. Thus, by fine-tuning the spectral intensity of individual LEDs, which emit different narrow bands of radiation, a white-light source characterized by a good colour-rendering index, $R_{a}$, and high luminous efficacy of radiation, $\eta_{\text {rad }}$, can be designed.

Creating a stable white light using multiple LEDs is a complex task since both colour rendering and luminous efficacy of the mixture depend on the emitted spectrum of the individual LEDs. Therefore, any change in the LED parameters (peak wavelengths, spectral widths, lumen outputs, etc.), for example, due to variations in junction temperature of the LEDs, causes a change in the spectrum of the LED devices and consequently a change in the spectrum of the white-light source $[7,8]$. The amount of change will depend on the magnitude of the deviations for each LED in the mixture. Keeping the mixture stable is further complicated by the fact that different types of LEDs are affected differently even when they are working under the same conditions [9]. Therefore, designing a simple yet accurate control system to maintain the white point within acceptable tolerances is one of the key challenges $[7,8,10,11]$.
We demonstrated that white-light sources based on LEDs have the potential of becoming the optimum choice for high-colour-rendering tasks [1], and in [2] we introduced our approach to intelligent spectral design. However, implementing a stable mixed-LED white-light source based on a theoretical (optimized) spectrum is an exacting task since there is no guarantee that a given set of real LEDs will exactly match the parameters used during the spectrum design. Hence, we have investigated the sensitivity of an optimized 4-band LED mixture to possible changes in the peak wavelengths of any one or more of the four LEDs. The aim was to quantify the influence of peak wavelength shifts in commercially available LEDs in terms of changes in correlated colour temperature, colour rendering, and luminous efficacy.

\section{Background}

2.1. Correlated Colour Temperature. Correlated colour temperature (CCT) of a light source is defined as the temperature of a Planckian (blackbody) radiator with chromaticity nearest to the chromaticity coordinates of the source on the CIE $1960(\mathrm{u}, \mathrm{v})$ diagram [12-14]. As the temperature of a blackbody radiator increases from $2000 \mathrm{~K}$ to $20000 \mathrm{~K}$, 
the perceived colour of the white light changes from "warm" (very reddish) to "cool" (very bluish). For example, incandescent lamps (circa $3000 \mathrm{~K}$ ) are characterized as being yellowish white, while fluorescent lights $(4000 \mathrm{~K}-7000 \mathrm{~K})$ are bluish white. In the text the correlated colour temperature is symbolized as $T_{c}$. Since a given change in colour temperature expressed in kelvins $(\mathrm{K})$ at different temperatures results in unequal changes in chromaticity [13], the colour temperature (or correlated colour temperature) is often expressed in reciprocal megakelvins, $\mathrm{MK}^{-1}$, and given the symbol $T_{\mathrm{cM}}$, where $T_{\mathrm{cM}}=10^{6} / T_{c[K]}$, which provides more uniform chromaticity differences. Reciprocal megakelvins were formally known as "microreciprocal degrees", abbreviated to "mireds." In the text the correlated colour temperature when expressed in Kelvin degrees is symbolized as $T_{c}$ and as $T_{\mathrm{cM}}$ when is expressed in $\mathrm{MK}^{-1}$.

2.2. Colour-Rendering Indices (CRI). Colour rendering is the characteristic of light sources that describes the visual effect of a light source on the colour of an object. Typically, at the present time, the colour-rendering properties of light sources are evaluated using the colour-rendering index $R_{a}$ [15]. In general, the higher the $R_{a}$ value of a light source, the more "natural" the colours of objects look under this source. Sunlight and incandescent lamps have $R_{a}=100$, which is the maximum value a light source can have. The acceptable value of CRI depends on the application of the light source. Values of $R_{a}$ above 80 are considered sufficient for most social and commercial indoor lighting applications, while $R_{a}$ above 90 or 95 is desirable in colour-matching tasks.

The fundamental idea behind the $R_{a}$ calculation is a comparison of the colours of eight standard test colour samples, which have low to moderate chromatic saturation (Table 1, samples 1-8), illuminated, in turn, by the test and reference light sources. The reference source has to be a source with the same CCT as the test source, either a Planckian radiator for test sources having CCT below $5000 \mathrm{~K}$ or a phase of daylight for test sources having CCT at or above $5000 \mathrm{~K}$. After accounting for chromatic adaptation with a Von Kries-type chromatic adaptation transform [13], the special colour-rendering index $R_{i}$ for each test colour sample is calculated as

$$
R_{i}=100-4.6 \Delta E_{i}, \quad i=1,2, \ldots, 8,
$$

where $\Delta E_{i}$ is the colour difference for each sample between the two light sources, as computed in CIE $1964 \mathrm{~W}^{*} \mathrm{U}^{*} \mathrm{~V}^{*}$ uniform colour space [13].

Averaging the eight $R_{i}$ values results in the general rendering index $R_{a}$ :

$$
R_{a}=\frac{1}{8} \sum_{i=1}^{8} R_{i}
$$

The CRI metric was originally designed to assess the quality of traditional fluorescent lamps and is found deficient when applied to assessing the colour-rendering quality of narrow-band light sources, such as the LED-based whitelight sources [16-20]. Hence, optimizing mixed-LED whitelight spectra using only eight colour samples of low to
TABLE 1: CIE test colours [15].

\begin{tabular}{lcc}
\hline No. & $\begin{array}{c}\text { Approximate munsell } \\
\text { notation }\end{array}$ & $\begin{array}{c}\text { Colour appearance under } \\
\text { daylight }\end{array}$ \\
\hline 1 & 7.5 R $6 / 4$ & Light greyish red \\
2 & 5 Y $6 / 4$ & Dark greyish yellow \\
Strong yellow green \\
3 & 5 GY 6/8 & Moderate yellowish green \\
4 & 2.5 G 6/6 & Light bluish green \\
5 & 10 BG 6/4 & Light Blue \\
6 & 5 PB 6/8 & Light violet \\
7 & 2.5 P 6/8 & Light reddish purple \\
8 & 10 P 6/8 & Strong red \\
9 & 4.5 R 4/13 & Strong yellow \\
10 & 5 Y 8/10 & Strong green \\
11 & 4.5 G 5/8 & Strong blue \\
12 & 3 PB 3/11 & Light human complexion \\
13 & 5 YR $8 / 4$ & Moderate olive green (leaf green) \\
14 & 5 GY 4/4
\end{tabular}

medium saturation can result in spectra with good computed $R_{a}$ but actually providing poor rendering of saturated colours. Therefore, in an attempt to improve the descriptive power of colour-rendering index, the number of test samples was extended to 14 by the addition of six additional test samples (Table 1, samples 9-14) representing saturated red, yellow, green, and blue, plus light human complexion and leaf green. Averaging the additional six $R_{i}$ values gives what we term index $R_{b}$, and averaging all $14 R_{i}$ values results in an "overall” index $R_{c}$.

In order to provide additional data on the colourrendering properties of each source, we have chosen also to quote the lowest $R_{i}\left(R_{i \min }\right)$ and the corresponding sample number $\left(i_{\min }\right)$ for the colour yielding $R_{i \min }$.

2.3. Radiant Luminous Efficacy. Radiant luminous efficacy, $\eta_{\text {rad }} \operatorname{lm} / \mathrm{W}$, compares the amounts of luminous flux and radiant flux emitted by the source:

$$
\eta_{\mathrm{rad}}=\frac{K_{m} \int_{\lambda} V(\lambda) S(\lambda) d \lambda}{\int_{\lambda} S(\lambda) d \lambda}
$$

where $S(\lambda)$ is the spectral distribution of the light source, $V(\lambda)$ is the CIE spectral sensitivity function for photopic vision, and $K_{m}$ is the maximum luminous efficacy of radiation $\left(K_{m}=683 \mathrm{~lm} / \mathrm{W}\right)$. The peak in the $V(\lambda)$ curve occurs for monochromatic radiation at $555 \mathrm{~nm}$.

\section{Method}

Evaluation of the influence of peak wavelengths on the characteristics of LED-based light sources was performed on a tetrachromatic spectrum of commercially available LEDs, chosen from the Luxeon range, with a peak wavelength combination of $470,530,590$, and $625 \mathrm{~nm}$. The mixture was optimized using a differential evolution algorithm [2]. The peak wavelengths were taken from the Luxeon datasheet 
TABLE 2: Ranges of peak wavelengths for four LEDs used to optimize the theoretical white-light source. The table is an extract from the Luxeon datasheet [21].

\begin{tabular}{lccc}
\hline LED & $\lambda_{i(\min )}(\mathrm{nm})$ & $\lambda_{i(\mathrm{typ})}(\mathrm{nm})$ & $\lambda_{i(\max )}(\mathrm{nm})$ \\
\hline Red $\left(\lambda_{R}\right)$ & $620.5(620)$ & $627(625)$ & 645 \\
Amber $\left(\lambda_{A}\right)$ & $584.5(585)$ & 590 & $597(595)$ \\
Green $\left(\lambda_{G}\right)$ & 520 & 530 & 550 \\
Blue $\left(\lambda_{B}\right)$ & 460 & 470 & 490 \\
\hline
\end{tabular}

[21] and are shown in Table 2 as $\lambda_{i(\text { typ })}$. The bracketed figures indicate the nearest available values in our data analysis which was based on a $5 \mathrm{~nm}$ wavelength interval. The optimized spectrum (Figure 1) has a correlated colour temperature $T_{c}$ of approximately $2919 \mathrm{~K}$, good colourrendering properties $\left(R_{a}=88\right)$, and high luminous efficacy $\left(\eta_{\mathrm{rad}}=390 \mathrm{~lm} / \mathrm{W}\right)$. This spectrum is the reference against which all later comparisons are made.

The Luxeon data specifies wavelength ranges $\lambda_{i}$ for the four LEDs in the mixture. The peak wavelength of the red LED can be anywhere between 620.5 and $645 \mathrm{~nm}$, the amber LED between 584.5 and $597 \mathrm{~nm}$, the green LED between 520 and $550 \mathrm{~nm}$, and 460 to $490 \mathrm{~nm}$ for the blue LED (Table 2). For the purpose of quantifying the influences of $\lambda_{i}$ values on the characteristics of the mixture, these ranges were divided into $5 \mathrm{~nm}$ bins $\lambda_{i j}$ (Table 3 ) and then the $T_{c}$, the colour-rendering indices $\left(R_{i}, R_{a}, R_{b}\right.$, and $\left.R_{c}\right)$, and the $\eta_{\text {rad }}$ values were calculated for all $882(6 \times 3 \times 7 \times 7)$ possible combinations of $\lambda_{i j}$. The first set of values was calculated at the $\lambda_{i j(\min )}=\lambda_{i 1}$ values and the last at the $\lambda_{i(\max )}$ values (i.e., $\left.\lambda_{R 6}, \lambda_{A 3}, \lambda_{G 7}, \lambda_{B 7}\right)$.

\section{Results}

4.1. Summary of Results for $T_{c}$. Table 4 summarizes the influences of each individual LED on the correlated colour temperature of the optimized tetrachromatic spectrum over the range of $\lambda_{i j}$, showing $T_{c i j}$ values in kelvins calculated for all $\lambda_{i j}$ bin values of one LED at a time, while the $\lambda_{k j}$ of other three LEDs were kept at their typical values, $\lambda_{k(\mathrm{typ})}, k \neq i$. For example, the $T_{c R j}$ values show the influence of changes in the red LED and were calculated with $\lambda_{A j}=\lambda_{A \text { (typ) }}=\lambda_{A 2}$, $\lambda_{G j}=\lambda_{G(\mathrm{typ})}=\lambda_{G 3}$, and $\lambda_{B j}=\lambda_{B(\mathrm{typ})}=\lambda_{B 3}$ and $\lambda_{R j}$ in the range from $\lambda_{R 1}$ to $\lambda_{R 6}$. Note that the typical peak wavelength of the red LED is $\lambda_{R(\text { typ })}=\lambda_{R 2}$. Thus, $T_{c R j}$ shows the dependence of $T_{c}$ on $\lambda_{R j}$, while other LEDs are at $\lambda_{k(\mathrm{typ})}, T_{c G j}$ shows the dependence of $T_{c}$ on $\lambda_{G j}$, while other LEDs are at $\lambda_{k(t y p) \text {, }}$ and so on. In addition, Table 4 lists the average values for each dependency of $T_{c}, T_{\text {ciavg }}$, and the difference between minimum and maximum values $\Delta T_{c i}=T_{c i \max }-T_{c i \min }$, the percentage change $\Delta T_{c i[\%]}=\Delta T_{c i} / T_{\text {ciavg }} \times 100$, and the differences in $\mathrm{MK}^{-1}$ defined as $\Delta T_{c i M}=10^{6} / T_{c i \min }-$ $10^{6} / T_{c i \max }$ and rounded to nearest integer.

As seen in Table 4, the changes in $\lambda_{R j}$ and $\lambda_{G j}$ result in the biggest $T_{c}$ variations (i.e., $\Delta T_{c \mathrm{RM}}=79 \mathrm{MK}^{-1}$ and $\Delta T_{c \mathrm{GM}}=70 \mathrm{MK}^{-1}$, resp.), while the blue LED causes the smallest variations $\left(\Delta T_{c B M}=4 \mathrm{MK}^{-1}\right)$. The correlated colour

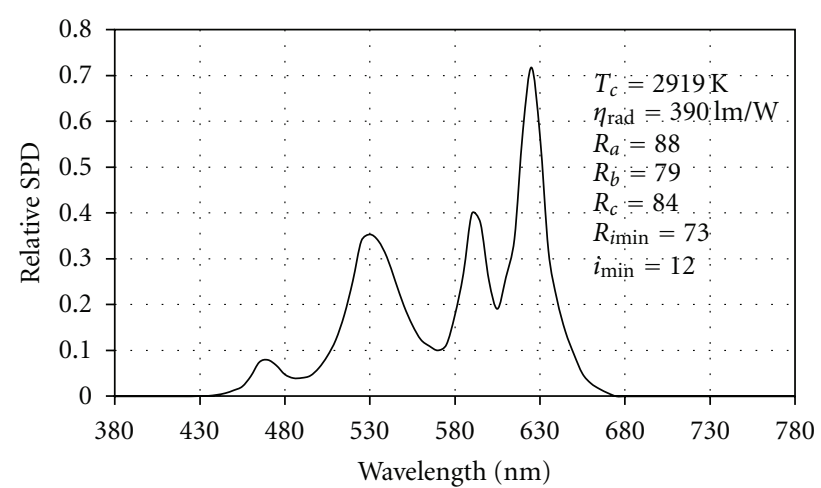

FIgURE 1: The spectrum of the optimized light source (used as reference for comparisons).

temperature of the tetrachromatic spectrum increases with $\lambda_{R j}$ and $\lambda_{B j}$ and decreases with increasing $\lambda_{G j}$ and $\lambda_{A j}$. It is evident that significant correlated colour temperature errors occur due to peak wavelength shifts in the red and green spectra and that the error caused by the shifts in the blue LED is one order of magnitude smaller.

The $T_{c}$ values are plotted in Figure 2. The five stepchanges in the $T_{c}$ plot are caused by a change of $\lambda_{R j}$ from bin to bin $(j \in[1,6])$ together with "reset" of other LEDs' peak wavelengths to their minimum values, that is, $\lambda_{k(\max )} \Rightarrow$ $\lambda_{k(\min )}=\lambda_{k 1}, k \neq i$. There are six distinctive patterns in the $T_{c}$ plot, one for each $\lambda_{R j}$ shift (bin). A $\lambda_{R j}$ pattern is influenced by seven green shifts $\left(\lambda_{G 1}-\lambda_{G 7}\right)$, and each green pattern is influenced by the seven blue peak wavelength shifts $\left(\lambda_{B 1}-\lambda_{B 7}\right)$. There are three $\lambda_{A j}$ shifts per a $\lambda_{B j}$ bin. As seen in Figure 2, the wavelength shifts of the red LED to a higher value increase the correlated colour temperature of the spectrum. Close inspection of the plot reveals that the increases in $T_{c}$ are larger at the higher $\lambda_{R j}$ values; the change from $\lambda_{R 1}$ (A) to $\lambda_{R 2}$ (B) resulted in $\Delta T_{c}=T_{c R 2}-T_{c R 1}=$ $757 \mathrm{~K}\left(104 \mathrm{MK}^{-1}\right)$, while the change from $\lambda_{R 5}$ (C) to $\lambda_{R 6}$ (D) resulted in $\Delta T_{c}=T_{c R 6}-T_{c R 5}=1256 \mathrm{~K}\left(116 \mathrm{MK}^{-1}\right)$. The influences of $\lambda_{i j}$ values on the $T_{c}$ of the mixture are further explored in Figures 3-5 and Table 5.

Keeping the red, green, and blue LED at their $\lambda_{k(\text { typ })}$ while changing $\lambda_{A j}$ from bin to bin $(j=1,2,3)$ resulted in $T_{c}$ having a maximum value $\left(T_{C A 1}=3048 \mathrm{~K}\right)$ at $\lambda_{A 1}$ which decreases to its minimum $\left(T_{C A 3}=2813 \mathrm{~K}\right)$ at $\lambda_{A 3}$ (Table 4$)$, a change of $235 \mathrm{~K}\left(27 \mathrm{MK}^{-1}\right)$. Figure 3 shows the dependency of the correlated colour temperature change caused by the $\lambda_{A j}$ shifts from $\lambda_{A 1}$ to $\lambda_{A 3}, \Delta T_{C M(A)}=T_{c M A 1}-T_{C M A 3}$, and Table 5 summarizes the influence of $\lambda_{R j}$ and $\lambda_{G j}$ on $\Delta T_{\mathrm{cM}(A)}$.

The $\Delta T_{\mathrm{CM}(\mathrm{A})}$ values are calculated as the red peak wavelengths change from $\lambda_{R j}$ to $\lambda_{R j+1}$ with the peak wavelength of the green LED being at a $\lambda_{G j}$ value, $j \in[1,7]$. The process was repeated for all $\lambda_{G j}$ and $\lambda_{R j}$ shifts. In Figure $3 \lambda_{R 2}, \lambda_{R 3}$, $\lambda_{R 4}$, and $\lambda_{G 1}$ to $\lambda_{G 7}$ are labelled for reference. It is observed that the $\Delta T_{\mathrm{CM}(\mathrm{A})}$ magnitudes of the discontinuities at the $\lambda_{R j}$ to $\lambda_{R j+1}$ increase at higher $\lambda_{R j}$ values (e.g., compare A and B). Table 5 reveals that the $\Delta T_{\mathrm{cM}(\mathrm{A})}$ values at $\lambda_{G 7}$ are lower than at $\lambda_{G 1}$ for $\lambda_{R j}=\left\{\lambda_{R 1}, \ldots, \lambda_{R 5}\right\}$. 
TABLE 3: The ranges of possible $\lambda_{i j}$ values divided into $5 \mathrm{~nm}$ bins, where $i$ designates one of four LEDs studied in this paper (red $\lambda_{R}$, amber $\lambda_{A}$, green $\lambda_{G}$, blue $\lambda_{B}$ ) and $j$ one of the possible $5 \mathrm{~nm}$ bins.

\begin{tabular}{|c|c|c|c|c|c|c|c|}
\hline LED & $\lambda_{i 1}(\mathrm{~nm})$ & $\lambda_{i 2}(\mathrm{~nm})$ & $\lambda_{i 3}(\mathrm{~nm})$ & $\lambda_{i 4}(\mathrm{~nm})$ & $\lambda_{i 5}(\mathrm{~nm})$ & $\lambda_{i 6}(\mathrm{~nm})$ & $\lambda_{i 7}(\mathrm{~nm})$ \\
\hline$\lambda_{R}$ & 620 & 625 & 630 & 635 & 640 & 645 & - \\
\hline$\lambda_{A}$ & 585 & 590 & 595 & - & - & - & - \\
\hline$\lambda_{G}$ & 520 & 525 & 530 & 535 & 540 & 545 & 550 \\
\hline$\lambda_{B}$ & 460 & 465 & 470 & 475 & 480 & 485 & 490 \\
\hline
\end{tabular}

TABLE 4: The summary of influences of each individual LED on correlated colour temperature of the optimized tetrachromatic spectrum, $\lambda_{i j}$ changes from bin to bin, while $\lambda_{k j}=\lambda_{k(\text { typ })}, k \neq i$.

\begin{tabular}{lccccccccccc}
\hline$T_{c i j} / \lambda_{i j}$ & $\lambda_{i 1}(\mathrm{~nm})$ & $\lambda_{i 2}(\mathrm{~nm})$ & $\lambda_{i 3}(\mathrm{~nm})$ & $\lambda_{i 4}(\mathrm{~nm})$ & $\lambda_{i 5}(\mathrm{~nm})$ & $\lambda_{i 6}(\mathrm{~nm})$ & $\lambda_{i 7}(\mathrm{~nm})$ & $T_{c i a v g}(\mathrm{~K})$ & $\Delta T_{c i}(\mathrm{~K})$ & $\Delta T_{c i[\%]}$ & $\Delta T_{c i M}\left(\mathrm{MK} \mathrm{K}^{-1}\right)$ \\
\hline$T_{c R j}$ & 2826 & 2919 & 3056 & 3229 & 3428 & 3641 & - & 3183 & 815 & 26 & 79 \\
$T_{c A j}$ & 3048 & 2919 & 2813 & - & - & - & - & 2927 & 235 & 8 & 27 \\
$T_{c G j}$ & 2972 & 2964 & 2919 & 2841 & 2733 & 2604 & 2459 & 2785 & 513 & 18 & 70 \\
$T_{c B j}$ & 2911 & 2915 & 2919 & 2923 & 2928 & 2935 & 2944 & 2925 & 33 & 1 & 4 \\
\hline
\end{tabular}

TABLE 5: The influences of $\lambda_{R j}$ and $\lambda_{G j}$ on $\Delta T_{\mathrm{cM}(\mathrm{A})}\left[\mathrm{MK}^{-1}\right]$. The values are rounded to 2 d.p.

\begin{tabular}{lcccccc}
\hline$\lambda_{R j} / \lambda_{G j}$ & $\lambda_{R 1}$ & $\lambda_{R 2}$ & $\lambda_{R 3}$ & $\lambda_{R 4}$ & $\lambda_{R 5}$ & $\lambda_{R 6}$ \\
\hline$\lambda_{G 1}$ & 1.66 & 1.65 & 1.64 & 1.70 & 1.57 & 1.27 \\
$\lambda_{G 2}$ & 1.62 & 1.61 & 1.52 & 1.53 & 1.38 & 1.28 \\
$\lambda_{G 3}$ & 1.40 & 1.41 & 1.46 & 1.62 & 1.40 & 1.19 \\
$\lambda_{G 4}$ & 1.34 & 1.39 & 1.46 & 1.56 & 1.39 & 1.28 \\
$\lambda_{G 5}$ & 1.45 & 1.39 & 1.52 & 1.56 & 1.52 & 1.43 \\
$\lambda_{G 6}$ & 1.30 & 1.42 & 1.61 & 1.57 & 1.47 & 1.64 \\
$\lambda_{G 7}$ & 1.13 & 1.46 & 1.56 & 1.45 & 1.54 & 1.57 \\
\hline
\end{tabular}

The influence of $\lambda_{G j}$ and $\lambda_{B j}$ on $T_{c}$ is shown in Figures 4 and 5 , respectively. The $T_{c}$ values shown in Figure 4 were calculated with peak wavelengths at $\lambda_{R \mathrm{j}}, \lambda_{A 1}, \lambda_{B 1}$, and $\lambda_{G j}$, and those in Figure 5 with peak wavelengths at $\lambda_{R j}$, $\lambda_{A 1}, \lambda_{B j}$, and $\lambda_{G \mathrm{j}}$. The correlated colour temperature of the optimized tetrachromatic spectrum $T_{c}$ decreases as the $\lambda_{G j}$ value increases. This is most easily observed by inspecting the plot in Figure 4 . Seven $T_{c}$ values are calculated $\left(T_{c G 1}-T_{c G 7}\right)$ for each of the six red LED bins. For the sake of clarity, only one set of $T_{c G 1}$ to $T_{c G 7}$ are labeled on the plot (at $\lambda_{R 4}$ ). It is observed that $T_{c}$ is highest at the $\lambda_{G 1}$ wavelength $\left(T_{c G 1}\right)$ and lowest at $\lambda_{G 7}$ wavelength $\left(T_{c G 7}\right)$.

The influence of the $\lambda_{B j}$ on the correlated colour temperature is not so obvious. In Figure 5 the $T_{c}$ values are plotted for all possible combinations of $\lambda_{B j}$. The general trend is that $T_{c}$ increases with $\lambda_{B j}$. However, close inspection of the plot reveals that, for some combination of $\lambda_{i j}$ (at the higher values of $\lambda_{R j}$ (i.e., $\lambda_{R 5}$ and $\lambda_{R 6}$ ) and lower values of $\lambda_{G j}$ (i.e., $\lambda_{G 1}$ and $\left.\lambda_{G 2}\right)$ ), the $T_{c}$ values exhibit a small drop in values instead of the increase observed elsewhere. This can be seen inspecting the circled part in Figure 5.

The collated results show that a change in $\lambda_{i j}$ could cause the correlated colour temperature, $T_{c}$, of the optimized mixture to be as high as $3976 \mathrm{~K}$ when $\lambda_{R j}=\lambda_{R 6}, \lambda_{A j}=$ $\lambda_{A 1}, \lambda_{G j}=\lambda_{G 1}$, and $\lambda_{B j}=\lambda_{B 1}$ and as low as $2311 \mathrm{~K}$ at minimum peak wavelengths for the red and blue LEDs and maximum peak wavelength values for the green and amber LEDs $\left(\lambda_{R 1}, \lambda_{A 3}, \lambda_{G 7}, \lambda_{B 1}\right)$ or a difference of $1665 \mathrm{~K}$ $\left(181 \mathrm{MK}^{-1}\right)$. The average value of $T_{c}$ was $3039 \mathrm{~K}$ with a standard deviation of $359 \mathrm{~K}$. Using the specified set of LEDs, $T_{c}$ changes $-21 \%$ to $+36 \%$ of its value in the optimized mixture.

4.2. Summary of Results for Colour-Rendering Indices. Table 6 provides a summary of the effects of peak wavelength shifts $\lambda_{i j}$ on the colour characteristics of the optimized tetrachromatic spectrum, expressed in terms of colourrendering indices $R_{a}, R_{b}$, and $R_{c}$. The errors for each colourrendering index, shown in Table 6, are calculated as $\Delta R=$ $R_{(\max )}-R_{(\min )}$ and $\Delta R_{[\%]}=\Delta R / R_{(\operatorname{avg})} \times 100$. It is seen that variations in colour-rendering characteristics are significant. In particular, the $R_{b}$ index is the most affected with $R_{b \max }=$ $84\left(\lambda_{R 2}, \lambda_{A 1}, \lambda_{G 2}, \lambda_{B 1}\right)$ and $R_{b \min }=31\left(\lambda_{R 1}, \lambda_{A 1}, \lambda_{G 7}, \lambda_{B 1}\right)$. These rather high variations $\left(\Delta R_{b}=53\right)$ mean that the colour rendering of the saturated colour samples will be poor. The most affected samples were Samples 9 (strong red) and 12 (strong blue); $R_{12}$ had the lowest $R_{i} 397$ times (in $45 \%$ of combinations) and $R_{9} 301$ times (in 34\% of combinations). Four more samples had the lowest $R_{i}$ : sample 11 (strong green) 142 times (in 16\% of combinations), sample 10 (strong yellow) 38 times (4\%), sample 3 (strong yellow green) three times, and sample 4 (moderate yellowish green) once. Significant changes were observed in $R_{i \min }=$ $\min \left\{R_{i}\right\}$, with $R_{i \text { min }}$ being always below its optimized value $R_{i \min (\text { opt })}=73$.

It is also interesting to note the effect of the peak wavelengths of four LEDs on the colour-rendering characteristics as shown in Figure 6. For the sake of clarity, the $R_{c}$ values $\left(R_{c}=R_{a}+R_{b}\right)$ are not plotted. As expected, there are five discontinuities in the plot, caused by a change of $\lambda_{R j}$ from bin 


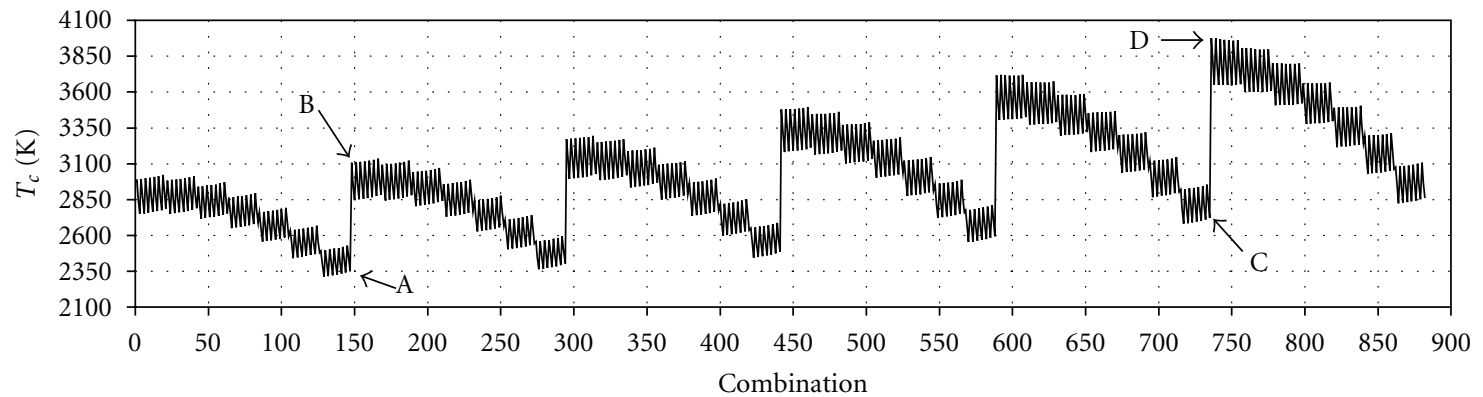

FIGURE 2: $T_{c}$ values calculated for the 882 possible combinations of $\lambda_{i j}$ values.

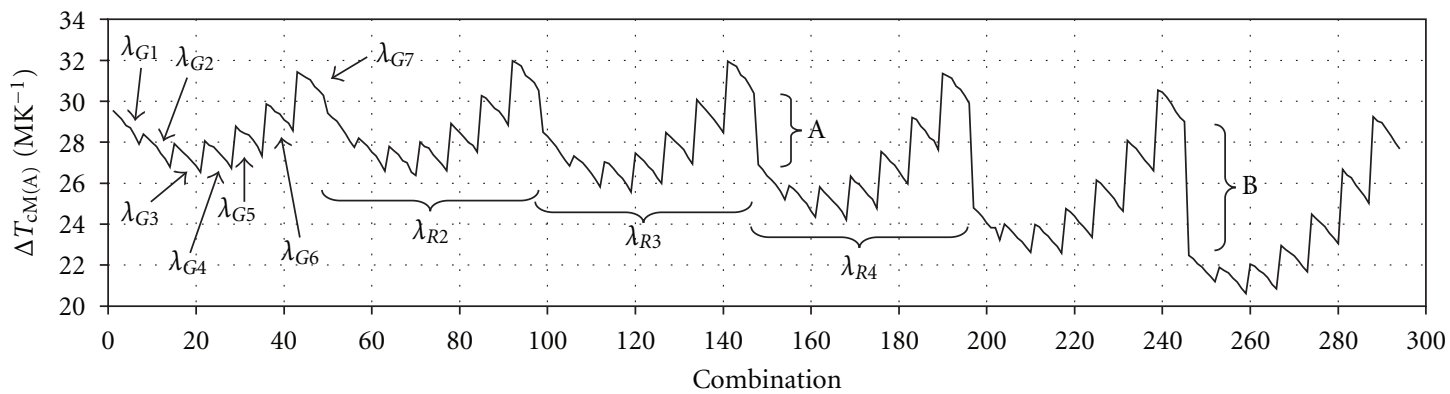

Figure 3: $\Delta T_{\mathrm{cM}(\mathrm{A})}=f\left(\lambda_{R j}, \lambda_{G j}, \lambda_{B j}\right)$.

TABLE 6: Summary of the influences of $\lambda_{i j}$ on the colour-rendering characteristics $\left(R_{a}, R_{b}, R_{c}\right)$ of the optimized tetrachromatic spec$\operatorname{trum}\left(R_{a(\mathrm{opt})}=88, R_{b(\mathrm{opt})}=79, R_{c(\mathrm{opt})}=84, R_{i \min (\mathrm{opt})}=73\right)$.

\begin{tabular}{lccccc}
\hline & $R_{(\text {avg })}$ & $R_{(\max )}$ & $R_{(\min )}$ & $\Delta R$ & $\Delta R_{[\%]}$ \\
\hline$R_{a}$ & 76 & 92 & 57 & 35 & 46 \\
$R_{b}$ & 64 & 84 & 31 & 53 & 83 \\
$R_{c}$ & 71 & 87 & 47 & 40 & 57 \\
$R_{i \min }$ & 30 & 73 & -45 & 118 & 387 \\
\hline
\end{tabular}

to bin together with "reset" of other LEDs' peak wavelengths to their minimum values $\left(\lambda_{k j(\min )}=\lambda_{k 1}, k \neq R\right)$. The largest $R_{b}$ change is when $\lambda_{R j}$ changes from $\lambda_{R 1}(A)$ to $\lambda_{R 2}(B)$, $\Delta R_{b}=45.5$. The size of $\Delta R_{b}$ decreases to $\Delta R_{b}=31.2$ at the $\lambda_{R 4}(C)$ to $\lambda_{R 5}(D)$ change, before increasing to 35.6 at the $\lambda_{R 5}$ $(E)$ to $\lambda_{R 6}(F)$ change.

4.3. Summary of Results for Radiant Luminous Efficacy. The effect of $\lambda_{i j}$ changes on $\eta_{\text {rad }}$ is evident in Figure 7 . The radiant efficacy has a maximum $\eta_{\mathrm{rad}(\max )}=438 \mathrm{~lm} / \mathrm{W}\left(\lambda_{R 1}, \lambda_{G 7}, \lambda_{B 7}\right.$, $\left.\lambda_{A 1}\right)$ at $\mathrm{A}$ and a minimum $\eta_{\mathrm{rad}(\min )}=308 \mathrm{~lm} / \mathrm{W}\left(\lambda_{R 6}, \lambda_{G 1}\right.$, $\lambda_{B 1}, \lambda_{A 3}$ ) at $B$ (which is $+12 \%$ and $-21 \%$ from the value in the optimized mixture $\left.\eta_{\mathrm{rad}(\mathrm{opt})}\right)$. The $\eta_{\mathrm{rad}}>\eta_{\mathrm{rad}(\mathrm{opt})}$ in 292 combinations of $\lambda_{i j}$ (in $33 \%$ of combinations) and was below $\eta_{\text {rad(opt) }} 590$ times (in $67 \%$ of combinations). Figure 7 also shows the step changes in $\eta_{\text {rad }}$ that result from $\lambda_{R j}$ changing from bin to bin (e.g., at $A$ in the graph).

The influences of the green, blue, and amber LEDs are further explored in Figure 8 , where $\eta_{\text {rad }}$ is plotted for $\lambda_{R 1}$ and $\lambda_{i j}$ of the other three LEDs changing from bin to bin. There are seven plots, one per each of seven $\lambda_{G j}$ bins and 21 $\eta_{\text {rad }}$ values (for different $\lambda_{A j}$ and $\lambda_{B j}$ combinations) per each $\lambda_{G j}$ bin. For the sake of clarity, the $\eta_{\text {rad }}$ points were marked only on the $\lambda_{G 1}$ plot. It is evident that the wavelength shifts of the green LED to a higher value $\lambda_{G j}$ result in a $\eta_{\text {rad }}$ increase; however, the increases are smaller at the higher values of $\lambda_{G j}$. It is also evident that, as $\lambda_{A j}$ changes from its lowest value (at $\lambda_{A 1}$ ) to its highest value (at $\lambda_{A 3}$ ), one observes reductions in $\eta_{\text {rad }}$. Again for the sake of clarity, only one set of $\lambda_{A 1}$ to $\lambda_{A 3}$ values are labeled on the $\lambda_{G 1}$ plot. Increasing $\lambda_{B j}$ leads to improvements in $\eta_{\mathrm{rad}}$. The $\eta_{\mathrm{rad}}$ values at $\lambda_{B 1}$ wavelength are lower than the $\eta_{\text {rad }}$ values at $\lambda_{B 7}$. It was interesting to observe that the difference between the $\eta_{\operatorname{rad}(\min )}(\mathrm{A})$ and $\eta_{\operatorname{rad}(\max )}(\mathrm{B})$ values was constant $(67 \mathrm{~lm} / \mathrm{W})$ across all $\lambda_{G j}$ plots.

\section{Conclusion}

We have investigated the sensitivity of an optimized tetrachromatic LED spectrum to changes in the peak wavelengths, $\lambda_{i j}$, of individual commercially available LEDs. The sensitivity was quantified in terms of changes in correlated colour temperature, colour-rendering indices, and luminous efficacy calculated for 882 combinations of $\lambda_{i j}$. The results emphasize the fact that the influence of peak wavelength shifts in commercial LEDs on the characteristics of the LEDbased light sources cannot be ignored.

1The correlated colour temperature increases with increases in the wavelengths of the red and green components 


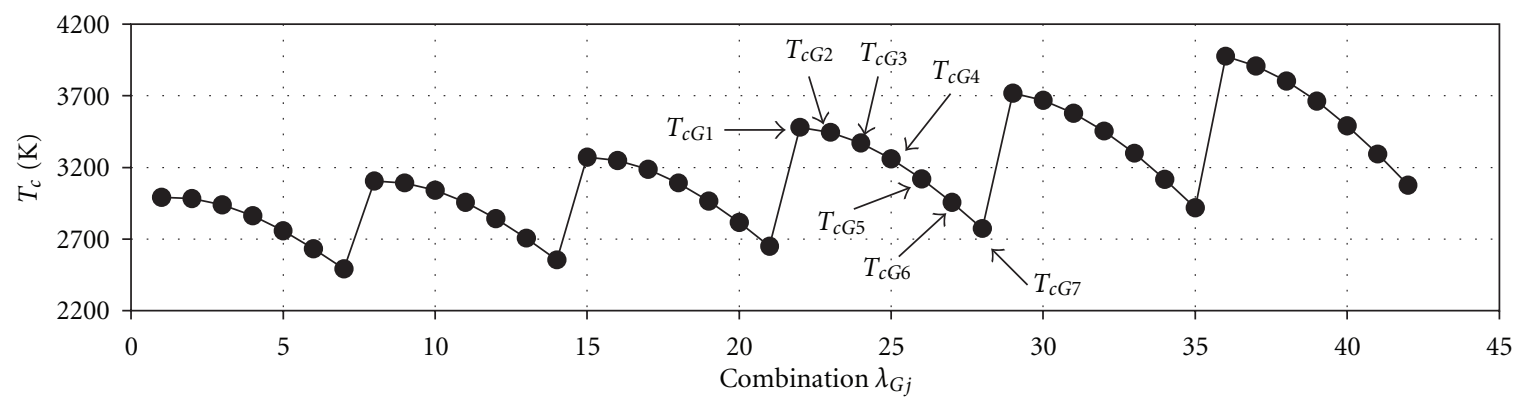

Figure 4: Influence of $\lambda_{G j}$ on $T_{c}$ of the optimized tetrachromatic spectrum; $\lambda_{G j} \uparrow \Rightarrow T_{c} \downarrow$.

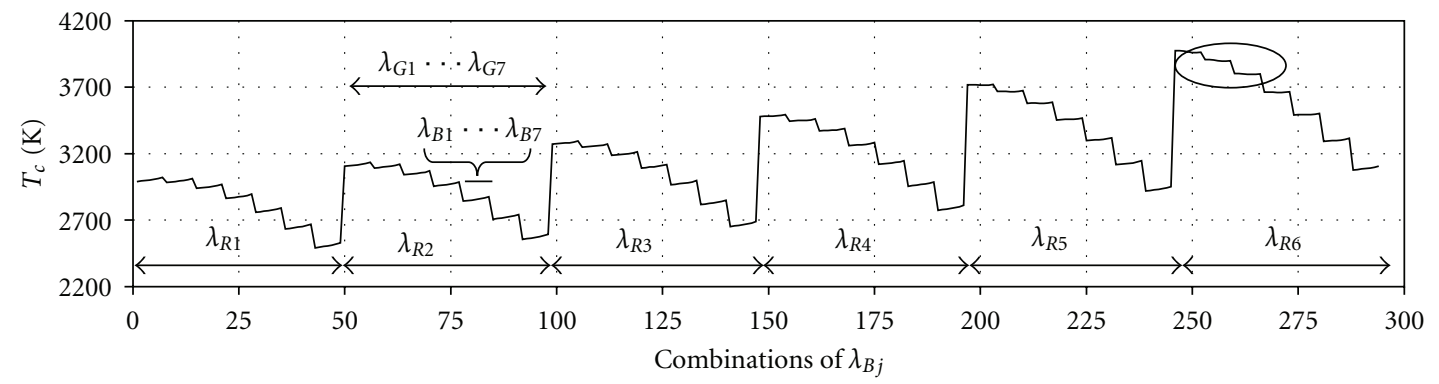

FIgURE 5: Influence of $\lambda_{B j}$ on $T_{c}$ of the optimized tetrachromatic spectrum. The circled portion of the graph shows that some values of $\lambda_{R j}$ and $\lambda_{G j}$ change the typical influence of $\lambda_{B j}$ (typical: $\lambda_{B j} \uparrow \Rightarrow T_{c} \uparrow$ ).

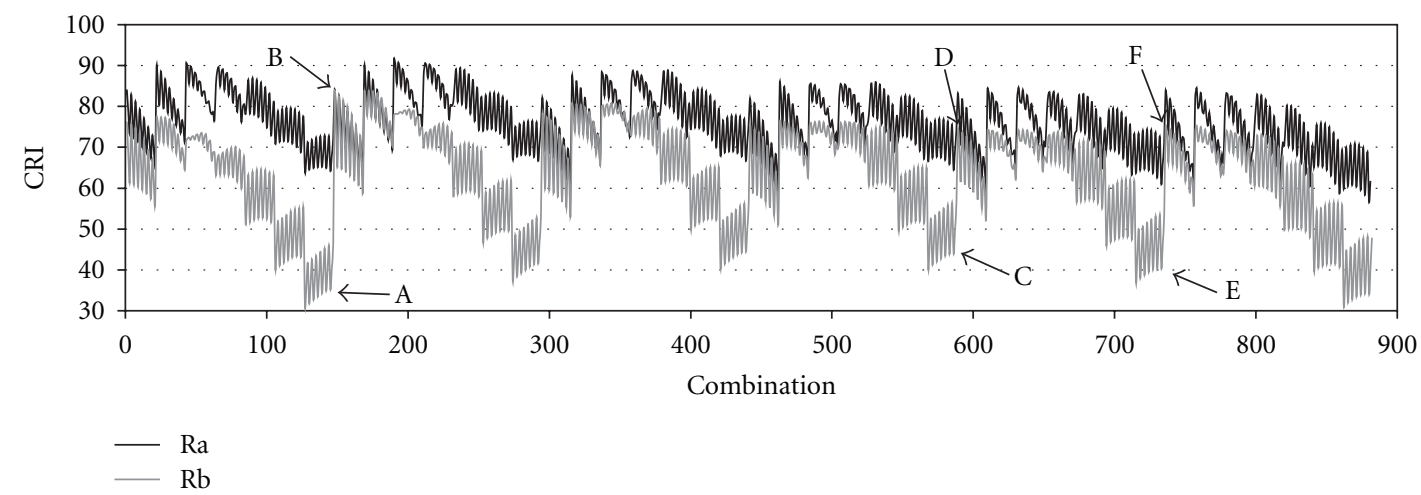

FIGURE 6: $R_{a}$ and $R_{b}$ values calculated for 882 possible combinations of $\lambda_{i j}$ values.

but decreases with increases in the wavelengths of green and amber components. The changes are significant. The correlated colour temperatures deviate $-21 \%$ and $+36 \%$ from the original value. The maximum $T_{c}$ of $3976 \mathrm{~K}$ was calculated when the red LEDs had the highest peak wavelength value and other LEDs were at their lowest values. The $T_{c}$ minimum was at minimum peak wavelengths for the red and blue LEDs and maximum peak wavelength values for the green and amber LEDs. Changes in correlated colour temperature caused by the deviation of the blue LED was one order of magnitude smaller than the changes caused by the other three LEDs. The largest $T_{c}$ deviations are caused by the red LED (26\%), followed by the green LED (18\%) for the wavelength ranges investigated.

Colour-rendering properties were also significantly affected by the variations in peak wavelengths, particularly the colour-rendering index $R_{b}$ which is determined by saturated test colour samples. The results for $R_{b}$ show a difference of 53 between its maximum and minimum values, $\Delta R_{b}=R_{b(\max )}-R_{b(\min )}=53$. These results make it clear that the colour rendering of strong colours can become very poor. The saturated test colour samples (red, blue, green, and yellow) had the lowest rendering index in $99.5 \%$ of all combinations. The $R_{a}$ index was less sensitive to the peak wavelength shifts, decreasing from $R_{a(\max )}=92$ to $R_{a(\min )}=57\left(\Delta R_{a}=35\right)$. These changes in colour rendering and correlated colour temperature are significant and can be problematic in numerous applications.

This study has also demonstrated that the radiant luminous efficacy, $\eta_{\text {rad }}$, of the optimized tetrachromatic LED spectrum changes significantly due to deviations in peak wavelength values. Its maximum value was $438 \mathrm{~lm} / \mathrm{W}$ and 


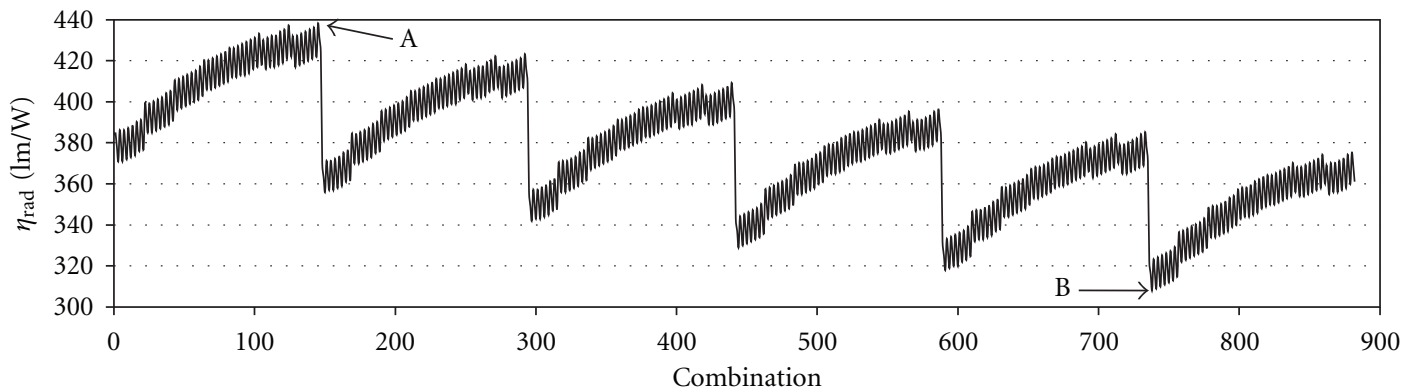

Figure 7: $\eta_{\text {rad }}$ values calculated for 882 possible combinations of $\lambda_{i j}$ values.

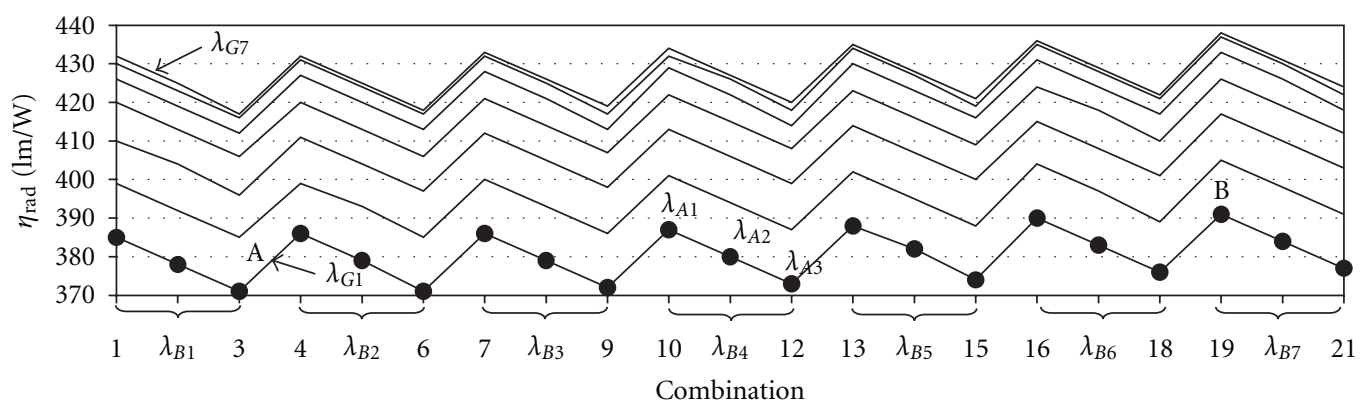

FIGURE 8: $\eta_{\text {rad }}=f\left(\lambda_{R 1}, \lambda_{i j}\right)$. There are seven plots, one per each $\lambda_{G j}$ bin, and $21 \eta_{\text {rad }}$ values for all possible combinations of $\lambda_{B j}$ and $\lambda_{A j}$ bins.

minimum was $308 \mathrm{~lm} / \mathrm{W}$. For $67 \%$ of combinations $\eta_{\mathrm{rad}}$ was below its optimized value $\eta_{\text {rad (opt) }}=390 \mathrm{~lm} / \mathrm{W}$. The $\eta_{\text {rad }}$ values decrease with the increase in red and amber peak wavelengths and increase with the blue and green wavelengths. This influence is opposite to the influence caused by the same set of LED variations on the correlated colour temperature.

A possible criticism of this study is that we have used the colour-rendering index, CRI, for the rendering of the white-light spectrum. While we are aware of the need for a new metric, CRI has been widely used for a number of years and is still the only officially recognized CIE method for assessing colour-rendering properties of light sources. We intend to adopt the new metric when formally adopted. In the meantime, we believe that it is appropriate to supplement $R_{a}$ with our additional ratings, $R_{b}, R_{c}$, and $R_{i \text { min }}$.

This study is only focused on the tetrachromatic spectrum of the LEDs chosen from the Luxeon range, and therefore these results cannot necessarily be extrapolated to other commercially available LEDs. Nevertheless, these results make it clear that the characteristics of mixed-LED white-light sources change significantly with changes in the peak wavelengths of individual LEDs.

\section{Acknowledgment}

The authors wish to acknowledge the support of this work by the RDTT Fund of the Manukau Institute of Technology and by the EET School of the Manukau Institute of Technology.

\section{References}

[1] A. Chalmers and S. Soltic, "Towards the optimum light source spectrum," Advances in OptoElectronics, vol. 2010, 9 pages, 2010.

[2] S. Soltic and A. N. Chalmers, "Differential evolution and its application to intelligent spectral design," in Proceedings of the The 16th Electronics New Zealand Conference (ENZCon '09), pp. 19-24, Dunedin, New Zealand, November 2009.

[3] A. N. Chalmers, C. Cuttle, L. Wang, and P. Luong, "The quest for high-colour-rendering high-luminous-efficacy light sources," in Proceedings of the The Illuminating Engineering Society of Australia and New Zealand Convention (IESANZ '07), Queenstown, New Zealand, 2007.

[4] Z. Lei, G. Xia, L. Ting, G. Xiaoling, L. Q. Ming, and S. Guangdi, "Color rendering and luminous efficacy of trichromatic and tetrachromatic LED-based white LEDs," Microelectronics Journal, vol. 38, pp. 1-6, 2007.

[5] Y. Ohno, "Simulation analysis of white LED spectra and color rendering," in Proceedings of the CIE Expert Symposium on LED Light Sources, Tokyo, Japan, 2004.

[6] A. Žakauskas, R. Vaicekauskas, F. Ivanauskas, R. Gaska, and M. S. Shur, "Optimization of white polychromatic semiconductor lamps," Applied Physics Letters, vol. 80, no. 2, pp. 234-236, 2002.

[7] S. Chhajed, Y. Xi, Y. L. Li, TH. Gessmann, and E. F. Schubert, "Influence of junction temperature on chromaticity and colorrendering properties of trichromatic white-light sources based on light-emitting diodes," Journal of Applied Physics, vol. 97, no. 5, Article ID 054506, pp. 1-8, 2005.

[8] S. Muthu, F. J. P. Schuurmans, and M. D. Pashley, "Red, green, and blue LEDs for white light illumination," IEEE Journal on Selected Topics in Quantum Electronics, vol. 8, no. 2, pp. 333$338,2002$. 
[9] N. Narendran, L. Deng, R. M. Pysar, Y. Gu, and H. Yu, "Performance characteristics of high-power light-emitting diodes," in Solid State Lighting, Proceedings of SPIE, pp. 267275, August 2003.

[10] X. Qu, S. C. Wong, and C. K. Tse, "Color control system for RGB LED light sources using junction temperature measurement," in Proceedings of the 33rd Annual Conference of the IEEE Industrial Electronics Conference (IECON '07), pp. 1363-1368, Taipei, Taiwan, November 2007.

[11] M. Dyble, N. Narendran, A. Bierman, and T. Klein, "Impact of dimming white LEDs: chromaticity shifts due to different dimming methods," in Solid State Lighting, vol. 5941 of Proceedings of SPIE, pp. 291-299, August 2005.

[12] R. S. Berns, Billmeyer and Saltzman's Principles of Color Technology, John Wiley \& Sons, New York, NY, USA, 3rd edition, 2000.

[13] G. Wyszecki and W. S. Stiles, Color Science: Concepts and Methods, Quantitative Data, and Formulae, John Wiley \& Sons, New York, NY, USA, 2nd edition, 2000.

[14] Commission Internationale de l'Eclairage, "Colorimetry," Tech. Rep. 15.3:2003, CIE Central Bureau, Vienna, Austria, 2003.

[15] Commission Internationale de l'Eclairage, "Method of measuring and specifying colour rendering properties of light sources," CIE Technical Report 13.3:1995, CIE Central Bureau, Vienna, Austria, 1995.

[16] Y. Ohno and W. Davis, "Rationale of color quality scale," LEDs Magazine, June 2010.

[17] W. Davis and Y. Ohno, "Toward an improved color rendering metric," in Solid State Lighting, vol. 5941 of Proceedings of SPIE, pp. 1-8, August 2005.

[18] E. Mahler, J. J. Ezrati, and F. Viénot, “Testing LED lighting for colour discrimination and colour rendering," Color Research and Application, vol. 34, no. 1, pp. 8-17, 2009.

[19] N. Narendran and L. Deng, "Color rendering properties of LED light sources," in Solid State Lighting II, Proceedings of SPIE, Seattle, Wash, USA, July 2002.

[20] N. Sándor and J. Schanda, "Visual colour rendering based on colour difference evaluations," Lighting Research and Technology, vol. 38, no. 3, pp. 225-239, 2006.

[21] Lumileds Lighting, "Luxeonß K2 Emitter," Technical Datasheet DS51, Lumileds Lighting US LLC., San Jose, Calif, USA, 2006. 

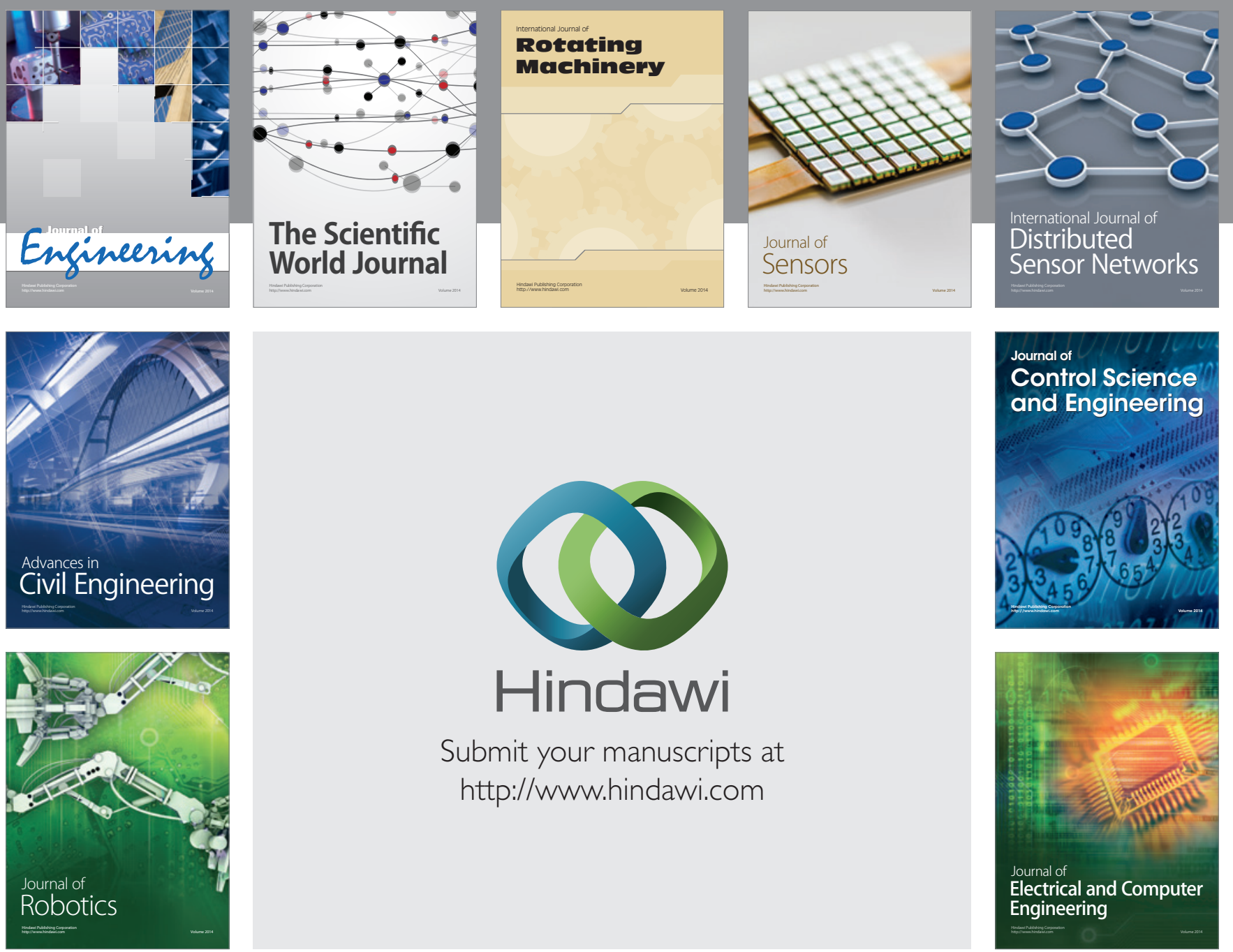

Submit your manuscripts at

http://www.hindawi.com
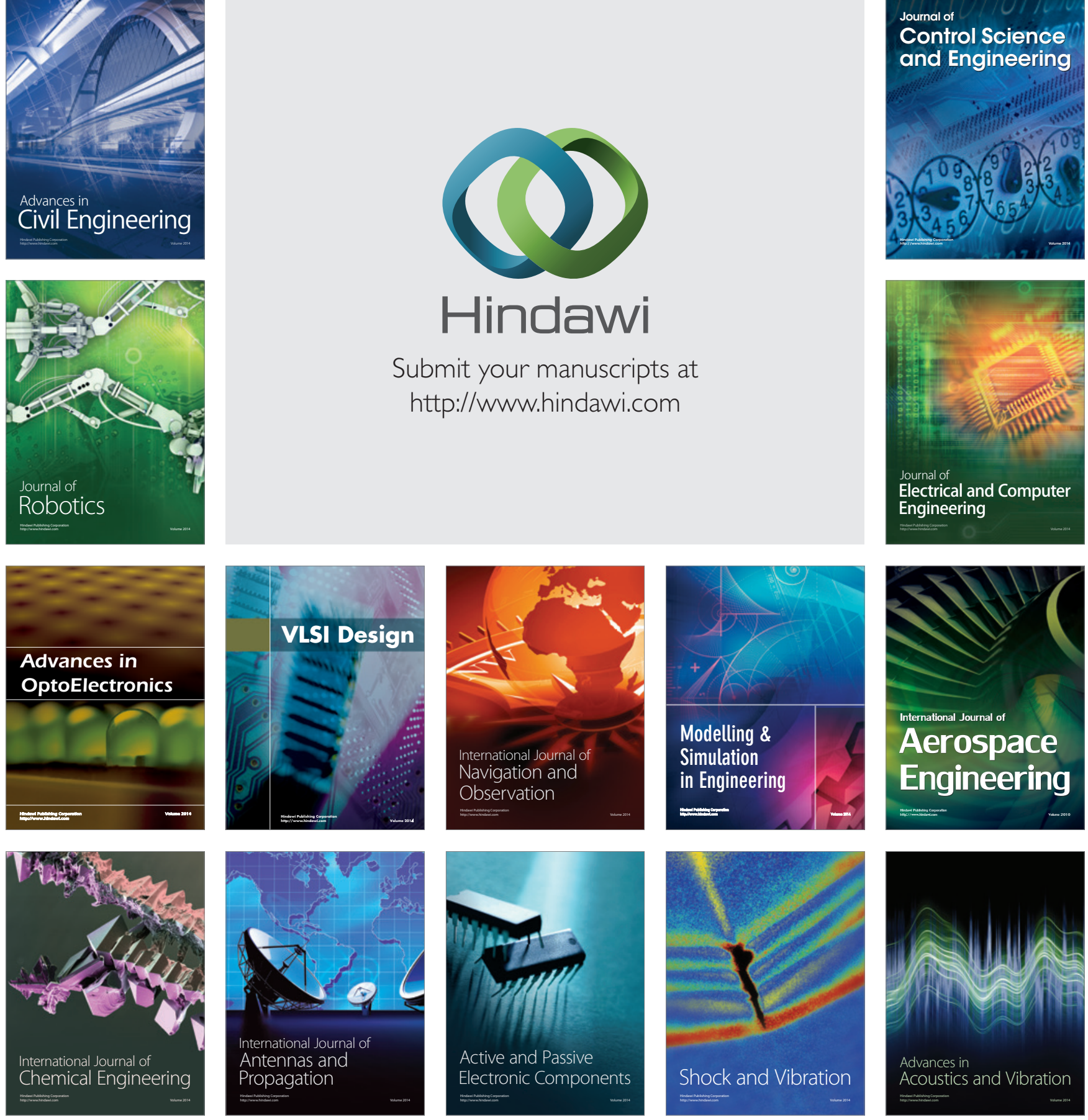